\title{
Note on the Invariance Properties of Operator Products Involving Generalized Inverses
}

\author{
Xiaoji Liu, ${ }^{1}$ Miao Zhang, ${ }^{1}$ and Yaoming $Y_{u^{2}}$ \\ ${ }^{1}$ College of Science, Guangxi University for Nationalities, Nanning 530006, China \\ ${ }^{2}$ School of Mathematical Sciences, Monash University, Melbourne, VIC 3800, Australia \\ Correspondence should be addressed to Xiaoji Liu; xiaojiliu72@126.com
}

Received 9 July 2013; Accepted 23 December 2013; Published 6 February 2014

Academic Editor: Jaan Janno

Copyright (c) 2014 Xiaoji Liu et al. This is an open access article distributed under the Creative Commons Attribution License, which permits unrestricted use, distribution, and reproduction in any medium, provided the original work is properly cited.

We investigate further the invariance properties of the bounded linear operator product $A C^{(1)} B^{(1)} D$ and its range with respect to the choice of the generalized inverses $X$ and $Y$ of bounded linear operators. Also, we discuss the range inclusion invariance properties of the operator product involving generalized inverses.

\section{Introduction}

Throughout this paper, by "an operator" we mean "a bounded linear operator over Hilbert spaces." Let the symbol $\mathscr{L}(\mathscr{H}, \mathscr{K})$ denote the set of all bounded linear operators from Hilbert space $\mathscr{H}$ to Hilbert space $\mathscr{K}$. In particular, $\mathscr{L}(\mathscr{H})=$ $\mathscr{L}(\mathscr{H}, \mathscr{H})$. For $A \in \mathscr{L}(\mathscr{H}, \mathscr{K})$, the symbols $A^{*}, \mathscr{R}(A)$, and $\mathscr{N}(A)$, respectively, denote its adjoint, range, and nullspace.

Recall that an operator $X \in \mathscr{L}(\mathscr{K}, \mathscr{H})$ is called the Moore-Penrose inverse of $A \in \mathscr{L}(\mathscr{H}, \mathscr{K})$ if $X$ satisfies the following operator equations:
(1) $A X A=A$;
(2) $X A X=X$;
(3) $(A X)^{*}=A X$;
(4) $(X A)^{*}=X A$.

If such an operator exists, then it is unique and is denoted by $A^{\dagger}$ (see, e.g., [1-4] for details). It is well known that the Moore-Penrose inverse $A^{\dagger}$ exists if and only if $\mathscr{R}(A)$ is closed. Let $i \in\{1,2,3,4\}$. If $X$ satisfies the equation $(i)$, then $X$ is an $\{i\}$-inverse of $A$ and is written as $X=A^{(i)}$. The set of all $\{i\}$ inverses of $A$ is denoted by $A\{i\}$. Obviously it is well defined that $A\{i, j\} \stackrel{\text { def }}{=} A\{i\} \cap A\{j\}, i, j \in\{1,2,3,4\}$ and write $X^{(i, j)}$ when $X \in A\{i, j\}$.

In 1971, Rao and Mitra [2] first discussed the invariance property of a matrix product $A B^{(1)} C$ with respect to any choice of $B^{(1)}$ and presented the necessary and sufficient conditions for such a matrix product to be invariant without respect to the choice of $B^{(1)}$. From then on, the invariance property has attracted more and more researchers to investigate it and showed its importance in theoretic research of many aspects, such as range invariance (see [5]), rank invariance (see [6]), invariance of the eigenvalues, singular values, and norms of matrix products (see [7]). The representational results, for example, have necessary and sufficient conditions for the invariance properties of $\mathscr{R}\left(A B^{(1)} C\right)$ (see [5]) and of rank $\left(A B^{(1)} C\right)$ (see [6]).

Recently in [8], the authors discussed the invariance of expressions of the matrix product $K L^{(1)} M^{(1)} N$. In [9], using the method of extremal ranks, the authors study the range inclusion invariance of the triple matrix product involving generalized inverses. And in [10], exploiting the matrix form of a bounded linear operator, the authors researched the invariance properties of the bounded linear operator product $T_{1} X T_{3}$ with respect to the choice of the generalized inverse $X$ of a bounded linear operator.

In this paper, we investigate further the invariance properties of the bounded linear operator product $A C^{(1)} B^{(1)} D$ and its range with respect to the choice of the generalized inverses $X$ and $Y$ of bounded linear operators. Also, we discuss the range inclusion invariance properties of the operator product involving generalized inverses. The paper is organized as follows. In Section 2, we introduce some lemmas. In Section 3, 
we present the equivalent conditions of the operator product $A C^{(1)} B^{(1)} D$ being invariant without respect to the choice of $B^{(1)}$ and $C^{(1)}$, which involve some inclusive relations among ranges of operators mentioned, the reverse order law for the $\{1\}$-inverses of $B$ and $C$, and $A C^{(1,2)} B^{(1,2)} D$ being invariant without respect to the choice of $B^{(1,2)}$ and $C^{(1,2)}$. And we also establish the relationship between invariance properties of $A C^{(1)} B^{(1)} D$ and its range under some condition. In Section 4 , we deduce the range inclusion invariance properties of the operator product involving $\{1\}$-inverses, $\{1,2\}$-inverses, and the some inclusive relations among ranges of operators mentioned.

\section{Lemmas}

In the section, we will introduce several lemmas as follows. The following lemma, with respect to generalized inverses of an operator, is similar to [1, Corollaries 2.1, 2.3, and 2.4], [2, Page 28], and $[9,(3.3)]$ for a matrix.

Lemma 1. Let $A \in \mathscr{L}(\mathscr{H}, \mathscr{K})$.

(i) If $A^{(1)} \in A\{1\}$, then

$$
\begin{aligned}
A\{1\}= & \left\{A^{(1)}+W-A^{(1)} A W A A^{(1)}: W \in \mathscr{L}(\mathscr{K}, \mathscr{H})\right\} \\
= & \left\{A^{(1)}+\left(I-A^{(1)} A\right) W_{1}\right. \\
& \left.+W_{2}\left(I-A A^{(1)}\right): W_{i} \in \mathscr{L}(\mathscr{K}, \mathscr{H}), i=1,2\right\} .
\end{aligned}
$$

(ii) Consider

$$
\begin{aligned}
& A\{1,2\}=\left\{X_{1} A X_{2}, X_{i} \in A\{1\}, i=1,2\right\} \\
&=\left\{\left[A^{\dagger}+\left(I-A^{\dagger} A\right) W_{1}\right] A\left[A^{\dagger}+W_{2}\left(I-A A^{\dagger}\right)\right]\right. \\
&\left.: W_{i} \in \mathscr{L}(\mathscr{K}, \mathscr{H}), i=1,2\right\} .
\end{aligned}
$$

(iii) If $A^{(1,3)} \in A\{1,3\}$, then $A\{1,3\}=\left\{A^{(1,3)}+(I-\right.$ $\left.\left.A^{(1,3)} A\right) W: W \in \mathscr{L}(\mathscr{K}, \mathscr{H})\right\}$.

(iv) If $A^{(1,4)} \in A\{1,4\}$, then $A\{1,4\}=\left\{A^{(1,4)}+W(I-\right.$ $\left.\left.A A^{(1,4)}\right): W \in \mathscr{L}(\mathscr{K}, \mathscr{H})\right\}$.

Lemma 2 (see [11, Lemmas 1.1 and 1.2]). Let $A \in \mathscr{L}(\mathscr{H}, \mathscr{K})$ have a closed range. Let $\mathscr{H}_{1}$ and $\mathscr{H}_{2}$ be closed and mutually orthogonal subspaces of $\mathscr{H}$, such that $\mathscr{H}=\mathscr{H}_{1} \oplus \mathscr{H}_{2}$. Let $\mathscr{K}_{1}$ and $\mathscr{K}_{2}$ be closed and mutually orthogonal subspaces of $\mathscr{K}$, such that $\mathscr{K}=\mathscr{K}_{1} \oplus \mathscr{K}_{2}$. Then the operator $A$ has the following matrix representations with respect to the orthogonal sums of subspaces $\mathscr{H}=\mathscr{H}_{1} \oplus \mathscr{H}_{2}=\mathscr{R}\left(A^{*}\right) \oplus \mathscr{N}(A)$ and $\mathscr{K}=\mathscr{R}(A) \oplus$ $\mathcal{N}\left(A^{*}\right)=\mathscr{K}_{1} \oplus \mathscr{K}_{2}$.

(i) Consider

$$
A=\left(\begin{array}{cc}
A_{1} & A_{2} \\
0 & 0
\end{array}\right):\left(\begin{array}{l}
\mathscr{H}_{1} \\
\mathscr{H}_{2}
\end{array}\right) \longrightarrow\left(\begin{array}{c}
\mathscr{R}(A) \\
\mathcal{N}\left(A^{*}\right)
\end{array}\right),
$$

where $D=A_{1} A_{1}^{*}+A_{2} A_{2}^{*}$ is invertible on $\mathscr{R}(A)$. Also,

$$
A^{\dagger}=\left(\begin{array}{ll}
A_{1}^{*} D^{-1} & 0 \\
A_{2}^{*} D^{-1} & 0
\end{array}\right) \text {. }
$$

(ii) Consider

$$
A=\left(\begin{array}{ll}
A_{1} & 0 \\
A_{2} & 0
\end{array}\right):\left(\begin{array}{c}
\mathscr{R}\left(A^{*}\right) \\
\mathcal{N}(A)
\end{array}\right) \longrightarrow\left(\begin{array}{c}
\mathscr{K}_{1} \\
\mathscr{K}_{2}
\end{array}\right)
$$

where $E=A_{1}^{*} A_{1}+A_{2}^{*} A_{2}$ is invertible on $\mathscr{R}\left(A^{*}\right)$. Also,

$$
A^{\dagger}=\left(\begin{array}{cc}
E^{-1} A_{1}^{*} & E^{-1} A_{2}^{*} \\
0 & 0
\end{array}\right) \text {. }
$$

In particular,

$$
A=\left(\begin{array}{cc}
A_{1} & 0 \\
0 & 0
\end{array}\right):\left(\begin{array}{c}
\mathscr{R}\left(A^{*}\right) \\
\mathscr{N}(A)
\end{array}\right) \longrightarrow\left(\begin{array}{c}
\mathscr{R}(A) \\
\mathscr{N}\left(A^{*}\right)
\end{array}\right),
$$

where $A_{1}$ is invertible. Moreover,

$$
A^{\dagger}=\left(\begin{array}{cc}
A_{1}^{-1} & 0 \\
0 & 0
\end{array}\right):\left(\begin{array}{c}
\mathscr{R}(A) \\
\mathcal{N}\left(A^{*}\right)
\end{array}\right) \longrightarrow\left(\begin{array}{c}
\mathscr{R}\left(A^{*}\right) \\
\mathcal{N}(A)
\end{array}\right) .
$$

The next lemma, with respect to generalized inverses of an operator, is similar to [8, Lemma 3] for matrices.

Lemma 3. Let $A \in \mathscr{L}(\mathscr{J}, \mathscr{K})$ and $B \in \mathscr{L}(\mathscr{H}, \mathscr{I})$ have closed ranges. Then $A W B=0$ for every $W \in \mathscr{L}(\mathscr{I}, \mathscr{J})$ if and only if $A=0$ or $B=0$.

Proof. If either $A$ or $B$ is zero, then $A W B=0$ for every $W \epsilon$ $\mathscr{L}(\mathscr{I}, \mathscr{J})$.

Assume $A, B \neq 0$. Then, by Lemma $2, A$ and $B$ can be represented, respectively, as the following matrix forms:

$$
\begin{gathered}
A=\left(\begin{array}{cc}
A_{11} & 0 \\
0 & 0
\end{array}\right):\left(\begin{array}{c}
\mathscr{R}\left(A^{*}\right) \\
\mathcal{N}(A)
\end{array}\right) \longrightarrow\left(\begin{array}{c}
\mathscr{R}(A) \\
\mathcal{N}\left(A^{*}\right)
\end{array}\right), \\
B=\left(\begin{array}{cc}
B_{11} & 0 \\
0 & 0
\end{array}\right):\left(\begin{array}{c}
\mathscr{R}\left(B^{*}\right) \\
\mathcal{N}(B)
\end{array}\right) \longrightarrow\left(\begin{array}{c}
\mathscr{R}(B) \\
\mathcal{N}\left(B^{*}\right)
\end{array}\right),
\end{gathered}
$$

where $A_{11}$ and $B_{11}$ are invertible in $\mathscr{L}\left(\mathscr{R}\left(A^{*}\right), \mathscr{R}(A)\right)$ and $\mathscr{L}\left(\mathscr{R}\left(B^{*}\right), \mathscr{R}(B)\right)$, respectively. Then take $0 \neq W_{11} \in$ $\mathscr{L}\left(\mathscr{R}(B), \mathscr{R}\left(A^{*}\right)\right)$ because $\mathscr{R}(B), \mathscr{R}\left(A^{*}\right) \neq 0$ and define

$$
W=\left(\begin{array}{cc}
W_{11} & 0 \\
0 & 0
\end{array}\right):\left(\begin{array}{c}
\mathscr{R}(B) \\
\mathcal{N}\left(B^{*}\right)
\end{array}\right) \rightarrow\left(\begin{array}{c}
\mathscr{R}\left(A^{*}\right) \\
\mathcal{N}(A)
\end{array}\right) .
$$

Thus if $A W B=0$, then $A_{11} W_{11} B_{11}=0$ and therefore $W_{11}=$ 0 because of the invertibility of $A_{11}$ and $B_{11}$. This leads to a contradiction. Hence $A W B \neq 0$ and then the result holds.

Lemma 4. Let $A \in \mathscr{L}(\mathscr{H}, \mathscr{K})$ have a closed range, $B \in$ $\mathscr{L}(\mathscr{H}, \mathscr{J})$. Then

$$
\mathscr{R}\left(B^{*}\right) \subseteq \mathscr{R}\left(A^{*}\right) \text { iff } B A^{\dagger} A=B .
$$

Proof. " $\Rightarrow$ " Since $\mathscr{R}(A)$ is closed, $A^{\dagger}$ exists and then $\mathscr{R}\left(B^{*}\right) \subseteq$ $\mathscr{R}\left(A^{*}\right)=\mathscr{R}\left(A^{\dagger} A\right)$. Thus, there exists an $X \in \mathscr{L}(J, H)$ such that $B^{*}=A^{\dagger} A X$. Hence $B=X^{*} A^{\dagger} A A^{\dagger} A=B A^{\dagger} A$.

" $\Leftarrow$ " Since $B^{*}=\left(B A^{\dagger} A\right)^{*}=A^{*}\left(B A^{\dagger}\right)^{*}, \mathscr{R}\left(B^{*}\right) \subseteq \mathscr{R}\left(A^{*}\right)$. 


\section{Invariance Properties of Operator Product}

In the section, we first present the main result of invariance properties.

Theorem 5. Let nonzero operators $A \in \mathscr{L}(\mathscr{I}, \mathscr{H}), B \in$ $\mathscr{L}(\mathscr{J}, \mathscr{K}), C \in \mathscr{L}(\mathscr{I}, \mathscr{J})$, and $D \in \mathscr{L}(\mathscr{H}, \mathscr{K})$ have closed ranges. Then the first three statements below are equivalent. Moreover, suppose that $A C^{\dagger} B^{\dagger} D$ has closed range. Then the following statements are equivalent:

(i) $A C^{(1)} B^{(1)} D$ does not depend on the choice of $C^{(1)} \in$ $C\{1\}$ and $B^{(1)} \in B\{1\}$;

(ii) $\mathscr{R}\left(A^{*}\right) \subseteq \mathscr{R}\left((B C)^{*}\right), \mathscr{N}(B) \subseteq \mathscr{R}(C), \mathscr{R}(D) \subseteq$ $\mathscr{R}(B C)$;

(iii) $A(B C)^{(1)} D$ does not depend on the choice of $(B C)^{(1)} \in$ $(B C)\{1\}$ and $C^{(1)} B^{(1)} \in(B C)\{1\}$ for every $C^{(1)} \in C\{1\}$ and $B^{(1)} \in B\{1\}$;

(iv) $\mathscr{R}\left(A C^{(1)} B^{(1)} D\right)$ does not depend on the choice of $C^{(1)} \in$ $C\{1\}$ and $B^{(1)} \in B\{1\}$.

Proof. (i) $\Rightarrow$ (ii): Since $A C^{(1)} B^{(1)} D$ does not depend on the choice of $C^{(1)} \in C\{1\}$ and $B^{(1)} \in B\{1\}$, we have

$$
A C^{(1)} B^{(1)} D=A C^{\dagger} B^{\dagger} D \text {. }
$$

Take $B^{(1)}=B^{\dagger}+U\left(I-B B^{\dagger}\right)$ and $C^{(1)}=C^{\dagger}+\left(I-C^{\dagger} C\right) V$, and put them into (13). Then $A\left[C^{\dagger}+\left(I-C^{\dagger} C\right) V\right]\left[B^{\dagger}+U(I-\right.$ $\left.\left.B B^{\dagger}\right)\right] D=A C^{\dagger} B^{\dagger} D$; namely,

$$
\begin{aligned}
& A C^{\dagger} U\left(I-B B^{\dagger}\right) D+A\left(I-C^{\dagger} C\right) V B^{\dagger} D \\
&+A\left(I-C^{\dagger} C\right) V U\left(I-B B^{\dagger}\right) D=0 .
\end{aligned}
$$

By the arbitrariness of $V$ and $U$,

$$
\begin{gathered}
A C^{\dagger} U\left(I-B B^{\dagger}\right) D=0, \quad A\left(I-C^{\dagger} C\right) V B^{\dagger} D=0, \\
A\left(I-C^{\dagger} C\right) V U\left(I-B B^{\dagger}\right) D=0 .
\end{gathered}
$$

By Lemma 3,

$$
\begin{gathered}
A C^{\dagger}=0 \quad \text { or } \quad\left(I-B B^{\dagger}\right) D=0, \\
A\left(I-C^{\dagger} C\right)=0 \quad \text { or } \quad B^{\dagger} D=0, \\
A\left(I-C^{\dagger} C\right)=0 \quad \text { or } \quad\left(I-B B^{\dagger}\right) D=0 .
\end{gathered}
$$

So if $\left(I-B B^{\dagger}\right) D \neq 0$, then $A C^{\dagger}=0$ and $A\left(I-C^{\dagger} C\right)=0$. Thus $A=0$, which contradicts $A \neq 0$. Similarly, $A\left(I-C^{\dagger} C\right) \neq 0$ implies $D=0$, which also leads to a contradiction. Hence

$$
A\left(I-C^{\dagger} C\right)=0, \quad\left(I-B B^{\dagger}\right) D=0 .
$$

Next take $B^{(1)}=B^{\dagger}+\left(I-B^{\dagger} B\right) U$ and $C^{(1)}=C^{\dagger}+V(I-$ $\left.C C^{\dagger}\right)$, and put them into (13). Then $A\left[C^{\dagger}+V\left(I-C C^{\dagger}\right)\right]\left[B^{\dagger}+\right.$ $\left.\left(I-B^{\dagger} B\right) U\right] D=A C^{\dagger} B^{\dagger} D$; namely,

$$
\begin{aligned}
A C^{\dagger} & \left(I-B^{\dagger} B\right) U D+A V\left(I-C C^{\dagger}\right) B^{\dagger} D \\
& +A V\left(I-C C^{\dagger}\right)\left(I-B^{\dagger} B\right) U D=0
\end{aligned}
$$

By the arbitrariness of $V$ and $U$ and Lemma 3,

$$
\begin{gathered}
A C^{\dagger}\left(I-B^{\dagger} B\right)=0, \quad\left(I-C C^{\dagger}\right) B^{\dagger} D=0, \\
\left(I-C C^{\dagger}\right)\left(I-B^{\dagger} B\right)=0 .
\end{gathered}
$$

By the above equation and (17),

$$
\begin{gathered}
A=A C^{\dagger} C=A C^{\dagger} B^{\dagger} B C, \quad D=B B^{\dagger} D=B C C^{\dagger} B^{\dagger} D, \\
\left(I-B^{\dagger} B\right)=C C^{\dagger}\left(I-B^{\dagger} B\right) .
\end{gathered}
$$

Namely, $\mathscr{R}\left(A^{*}\right) \subseteq \mathscr{R}\left((B C)^{*}\right), \mathscr{R}(D) \subseteq \mathscr{R}(B C), \mathcal{N}(B) \subseteq$ $\mathscr{R}(C)$.

(ii) $\Rightarrow$ (iii): The inclusion $\mathcal{N}(B) \subseteq \mathscr{R}(C)$ implies $I-B^{\dagger} B=$ $C C^{\dagger}\left(I-B^{\dagger} B\right)$ and then $I-B^{\dagger} B=\left(I-B^{\dagger} B\right) C C^{\dagger}$ by the definition of the Moore-Penrose inverse. So the two equations lead to $C C^{\dagger} B^{\dagger} B=B^{\dagger} B C C^{\dagger}$ and therefore $B C C^{\dagger}\left(I-B^{\dagger} B\right)=0$ and $\left(I-C C^{\dagger}\right) B^{\dagger} B C=0$. Consequently, for every $C^{(1)} \in C\{1\}$ and $B^{(1)} \in B\{1\}$,

$$
\begin{aligned}
B C C^{(1)} B^{(1)} B C= & B C\left[C^{\dagger}+\left(I-C^{\dagger} C\right) V_{1}+V_{2}\left(I-C C^{\dagger}\right)\right] \\
& \times\left[B^{\dagger}+\left(I-B^{\dagger} B\right) U_{1}+U_{2}\left(I-B B^{\dagger}\right)\right] B C \\
= & {\left[B C C^{\dagger}+B C V_{2}\left(I-C C^{\dagger}\right)\right] } \\
& \times\left[B^{\dagger} B C+\left(I-B^{\dagger} B\right) U_{1} B C\right] \\
= & B C C^{\dagger} B^{\dagger} B C=B C .
\end{aligned}
$$

That is, $C^{(1)} B^{(1)} \in(B C)\{1\}$.

Note that $\mathscr{R}\left(A^{*}\right) \subseteq \mathscr{R}\left((B C)^{*}\right)$ and $\mathscr{R}(D) \subseteq \mathscr{R}(B C)$ imply $A=X B C$ and $D=B C Y$ for certain operators $X, Y$, respectively. Then, by Lemma 1 ,

$$
\begin{aligned}
A(B C)^{(1)} D= & X B C \\
& \times\left[C^{\dagger} B^{\dagger}+\left(I-C^{\dagger} B^{\dagger} B C\right) U\right. \\
& \left.+V\left(I-B C C^{\dagger} B^{\dagger}\right)\right] B C Y \\
= & X B C C^{\dagger} B^{\dagger} B C Y=A C^{\dagger} B^{\dagger} D .
\end{aligned}
$$

That is, $A(B C)^{(1)} D$ does not depend on the choice of $(B C)^{(1)} \epsilon$ (BC) $\{1\}$.

(iii) $\Rightarrow(\mathrm{i})$ : It is obvious.

Now we consider the situation under $A C^{\dagger} B^{\dagger} D$ having closed range. It is evident that (i) $\Rightarrow$ (iv). We will show that (iv) $\Rightarrow$ (ii).

Obviously, $\mathscr{R}\left(A C^{(1)} B^{(1)} D\right)=\mathscr{R}\left(A C^{\dagger} B^{\dagger} D\right)$ holds for every $C^{(1)} \in C\{1\}$ and $B^{(1)} \in B\{1\}$. Then $A C^{(1)} B^{(1)} D$ has closed range and therefore

$$
\left(A C^{\dagger} B^{\dagger} D\right)\left(A C^{\dagger} B^{\dagger} D\right)^{\dagger}=\left(A C^{(1)} B^{(1)} D\right)\left(A C^{(1)} B^{(1)} D\right)^{\dagger} .
$$


Putting $C^{(1)}=C^{\dagger}+\left(I-C^{\dagger} C\right) V$ and $B^{(1)}=B^{\dagger}+U\left(I-B B^{\dagger}\right)$ into (23) yields

$$
\begin{aligned}
& \left(A C^{\dagger} B^{\dagger} D\right)\left(A C^{\dagger} B^{\dagger} D\right)^{\dagger} \\
& \quad=\left(A C^{\dagger} B^{\dagger} D+\Delta_{1}\right)\left(A C^{\dagger} B^{\dagger} D+\Delta_{1}\right)^{\dagger},
\end{aligned}
$$

where

$$
\begin{aligned}
\Delta_{1}= & A C^{\dagger} U\left(I-B B^{\dagger}\right) D+A\left(I-C^{\dagger} C\right) V B^{\dagger} D \\
& +A\left(I-C^{\dagger} C\right) V U\left(I-B B^{\dagger}\right) D .
\end{aligned}
$$

Putting $C^{(1)}=C^{\dagger}+V\left(I-C C^{\dagger}\right)$ and $B^{(1)}=B^{\dagger}+\left(I-B^{\dagger} B\right) U$ into (23) yields

$$
\begin{aligned}
& \left(A C^{\dagger} B^{\dagger} D\right)\left(A C^{\dagger} B^{\dagger} D\right)^{\dagger} \\
& \quad=\left(A C^{\dagger} B^{\dagger} D+\Delta_{2}\right)\left(A C^{\dagger} B^{\dagger} D+\Delta_{2}\right)^{\dagger},
\end{aligned}
$$

where

$$
\begin{aligned}
\Delta_{2}= & A C^{\dagger}\left(I-B^{\dagger} B\right) U D+A V\left(I-C C^{\dagger}\right) B^{\dagger} D \\
& +A V\left(I-C C^{\dagger}\right)\left(I-B^{\dagger} B\right) U D .
\end{aligned}
$$

By the arbitrariness of $V$ and $U,(24)$ and (26) lead to $\Delta_{i}=$ $0, i=1,2$. From these, as the argument in (i) $\Rightarrow$ (ii) above, we can reach (ii).

Note that $A C^{\dagger} B^{\dagger} D=0$ if and only if $\mathscr{R}\left(B^{\dagger} D\right) \subseteq \mathscr{N}\left(A C^{\dagger}\right)$. Thus, we have the following corollary.

Corollary 6. Let nonzero operators $A \in \mathscr{L}(\mathscr{I}, \mathscr{H}), B \in$ $\mathscr{L}(\mathscr{J}, \mathscr{K}), C \in \mathscr{L}(\mathscr{I}, \mathscr{J})$, and $D \in \mathscr{L}(\mathscr{H}, \mathscr{K})$ have closed ranges. Then the following statements are equivalent:

(i) $A C^{(1)} B^{(1)} D=0$ holds for every $C^{(1)} \in C\{1\}$ and $B^{(1)} \in$ $B\{1\}$;

(ii) $\mathscr{R}\left(A^{*}\right) \subseteq \mathscr{R}\left((B C)^{*}\right), \mathscr{N}(B) \subseteq \mathscr{R}(C), \mathscr{R}(D) \subseteq$ $\mathscr{R}(B C), \mathscr{R}\left(B^{\dagger} D\right) \subseteq \mathscr{N}\left(A C^{\dagger}\right)$;

(iii) $A(B C)^{(1)} D=0$ holds for every $(B C)^{(1)} \in(B C)\{1\}$ and $C^{(1)} B^{(1)} \in(B C)\{1\}$ for every $C^{(1)} \in C\{1\}$ and $B^{(1)} \epsilon$ $B\{1\}$.

Corollary 7 (see [10, Theorem 2.1]). Let nonzero operators $A \in \mathscr{L}(\mathscr{I}, \mathscr{H}), B \in \mathscr{L}(\mathscr{H}, \mathscr{K})$, and $C \in \mathscr{L}(\mathscr{H}, \mathscr{K})$ have closed ranges. Then the following statements are equivalent:

(i) the operator product $A B^{(1)} C$ does not depend on the choice of $B^{(1)} \in B\{1\}$;

(ii) $\mathscr{R}\left(A^{*}\right) \subseteq \mathscr{R}\left(B^{*}\right), \mathscr{R}(C) \subseteq \mathscr{R}(B)$.

When $A=B C=D$ in Theorem 5, we have the next result.

Corollary 8. Let nonzero operators $B \in \mathscr{L}(\mathscr{J}, \mathscr{K})$, and $C \in$ $\mathscr{L}(\mathscr{I}, \mathscr{J})$ have closed ranges. Then the following statements are equivalent: (i) $B C C^{(1)} B^{(1)} B C$ does not depend on the choice of $C^{(1)} \in$ $C\{1\}$ and $B^{(1)} \in B\{1\}$;

(ii) $\mathscr{N}(B) \subseteq \mathscr{R}(C)$;

(iii) $C^{(1)} B^{(1)} \in(B C)\{1\}$ for every $C^{(1)} \in C\{1\}$ and $B^{(1)} \in$ $B\{1\}$.

Next we will research the situation with respect to $\{1,2\}$ inverses.

Theorem 9. Let nonzero operators $A \in \mathscr{L}(\mathscr{I}, \mathscr{H}), B \in$ $\mathscr{L}(\mathscr{J}, \mathscr{K}), C \in \mathscr{L}(\mathscr{I}, \mathscr{J})$ and $D \in \mathscr{L}(\mathscr{H}, \mathscr{K})$ have closed ranges. Then the first three statements below are equivalent. Moreover, suppose that $A C^{\dagger} B^{\dagger} D$ has closed range. Then the following statements are equivalent:

(i) $A C^{(1,2)} B^{(1,2)} D$ does not depend on the choice of $C^{(1,2)} \in$ $C\{1,2\}$ and $B^{(1,2)} \in B\{1,2\}$;

(ii) $\mathscr{R}\left(A^{*}\right) \subseteq \mathscr{R}\left((B C)^{*}\right), \mathscr{N}(B) \subseteq \mathscr{R}(C), \mathscr{R}(D) \subseteq$ $\mathscr{R}(B C)$;

(iii) $A(B C)^{(1,2)} D$ does not depend on the choice of $(B C)^{(1,2)} \in(B C)\{1,2\}$ and $C^{(1,2)} B^{(1,2)} \in(B C)\{1\}$ for every $C^{(1,2)} \in C\{1,2\}$ and $B^{(1,2)} \in B\{1,2\}$;

(iv) $\mathscr{R}\left(A C^{(1,2)} B^{(1,2)} D\right)$ does not depend on the choice of $C^{(1,2)} \in C\{1,2\}$ and $B^{(1,2)} \in B\{1,2\}$.

Proof. (ii) $\Rightarrow(\mathrm{i}): \mathscr{R}\left(A^{*}\right) \subseteq \mathscr{R}\left((B C)^{*}\right)$ and $\mathscr{R}(D) \subseteq \mathscr{R}(B C)$ imply $A=W_{1} B C$ for some $W_{1}$ and $D=B C W_{2}$ for some $W_{2}$, respectively. By Lemma $1, C^{(1,2)}=X_{1} C X_{2}$ and $B^{(1,2)}=Y_{1} B Y_{2}$ where $X_{i} \in C\{1\}, Y_{i} \in B\{1\}, i=1,2$. So, by Theorem 5,

$$
\begin{aligned}
A C^{(1,2)} B^{(1,2)} D & =W_{1} B C X_{1} C X_{2} Y_{1} B Y_{2} B C W_{2} \\
& =W_{1} B C X_{2} Y_{1} B C W_{2} \\
& =A X_{2} Y_{1} D=A C^{\dagger} B^{\dagger} D
\end{aligned}
$$

and then (i) is true.

(i) $\Rightarrow$ (ii): Since $A C^{(1,2)} B^{(1,2)} D$ does not depend on the choice of $C^{(1,2)} \in C\{1,2\}$ and $B^{(1,2)} \in B\{1,2\}$, we have

$$
A C^{(1,2)} B^{(1,2)} D=A C^{\dagger} B^{\dagger} D \text {. }
$$

First of all, we will show $A C^{\dagger} \neq 0$ by contradiction. For this, assume $A C^{\dagger}=0$. Take $C^{(1,2)}=\left[C^{\dagger}+\left(I-C^{\dagger} C\right) V_{1}\right] C\left[C^{\dagger}+\right.$ $\left.V_{2}\left(I-C C^{\dagger}\right)\right]$. Then putting $A C^{(1,2)}=A V_{1} C\left[C^{\dagger}+V_{2}\left(I-C C^{\dagger}\right)\right]$ and $B^{(1,2)}=B^{\dagger} B\left[B^{\dagger}+U_{2}\left(I-B B^{\dagger}\right)\right]$ into (29) leads to

$$
A V_{1} C\left[C^{\dagger}+V_{2}\left(I-C C^{\dagger}\right)\right] B^{\dagger} B\left[B^{\dagger}+U_{2}\left(I-B B^{\dagger}\right)\right] D=0 .
$$

Namely,

$$
\begin{aligned}
0= & A V_{1} C C^{\dagger} B^{\dagger} D+A V_{1} C V_{2}\left(I-C C^{\dagger}\right) B^{\dagger} D \\
& +A V_{1} C C^{\dagger} B^{\dagger} B U_{2}\left(I-B B^{\dagger}\right) D \\
& +A V_{1} C V_{2}\left(I-C C^{\dagger}\right) B^{\dagger} B U_{2}\left(I-B B^{\dagger}\right) D .
\end{aligned}
$$


By the arbitrariness of $V_{1}, V_{2}$, and $U_{2}$, the condition that $A \neq 0$ and $C \neq 0$, and Lemma 3 ,

$$
\begin{gathered}
C C^{\dagger} B^{\dagger} D=0, \\
\left(I-C C^{\dagger}\right) B^{\dagger} D=0, \\
C C^{\dagger} B^{\dagger} B=0 \quad \text { or } \quad\left(I-B B^{\dagger}\right) D=0, \\
\left(I-C C^{\dagger}\right) B^{\dagger} B=0 \quad \text { or } \quad\left(I-B B^{\dagger}\right) D=0 .
\end{gathered}
$$

By (32), $B^{\dagger} D=0$ and then $\left(I-B B^{\dagger}\right) D \neq 0$ since $D \neq 0$. Thus, by (33), $B^{\dagger} B=0$ and then $B=0$, which contradicts $B \neq 0$. Hence $A C^{\dagger} \neq 0$.

Now take $B^{(1,2)}=\left[B^{\dagger}+\left(I-B^{\dagger} B\right) U_{1}\right] B\left[B^{\dagger}+U_{2}\left(I-B B^{\dagger}\right)\right]$ and $C^{(1,2)}=C^{\dagger}$, and put them into (29). Then

$$
\begin{gathered}
A C^{\dagger} B^{\dagger} B U_{2}\left(I-B B^{\dagger}\right) D+A C^{\dagger}\left(I-B^{\dagger} B\right) U_{1} B B^{\dagger} D \\
+A C^{\dagger}\left(I-B^{\dagger} B\right) U_{1} B U_{2}\left(I-B B^{\dagger}\right) D=0 .
\end{gathered}
$$

By the arbitrariness of $U_{1}$ and $U_{2}$ and Lemma 3,

$$
\begin{gathered}
A C^{\dagger} B^{\dagger} B=0 \quad \text { or } \quad\left(I-B B^{\dagger}\right) D=0, \\
A C^{\dagger}\left(I-B^{\dagger} B\right)=0 \quad \text { or } \quad B B^{\dagger} D=0, \\
A C^{\dagger}\left(I-B^{\dagger} B\right)=0 \quad \text { or } \quad\left(I-B B^{\dagger}\right) D=0 .
\end{gathered}
$$

So if $\left(I-B B^{\dagger}\right) D \neq 0$, then $A C^{\dagger} B^{\dagger} B=0$ and $A C^{\dagger}\left(I-B^{\dagger} B\right)=0$. Thus $A C^{\dagger}=0$, which contradicts $A C^{\dagger} \neq 0$. Similarly, $A C^{\dagger}(I-$ $\left.B^{\dagger} B\right) \neq 0$ implies $D=0$, which also leads to a contradiction. Hence

$$
A C^{\dagger}\left(I-B^{\dagger} B\right)=0, \quad\left(I-B B^{\dagger}\right) D=0 .
$$

Next take $B^{(1,2)}=B^{\dagger}$ and $C^{(1,2)}=\left[C^{\dagger}+\left(I-C^{\dagger} C\right) V_{1}\right] C\left[C^{\dagger}+\right.$ $\left.V_{2}\left(I-C C^{\dagger}\right)\right]$, and put them into (29). Then

$$
\begin{gathered}
A C^{\dagger} C V_{2}\left(I-C C^{\dagger}\right) B^{\dagger} D+A\left(I-C^{\dagger} C\right) V_{1} C C^{\dagger} B^{\dagger} D \\
+A\left(I-C^{\dagger} C\right) V_{1} C V_{2}\left(I-C C^{\dagger}\right) B^{\dagger} D=0 .
\end{gathered}
$$

By the arbitrariness of $V_{1}$ and $V_{2}$ and Lemma 3,

$$
\begin{gathered}
A C^{\dagger} C=0 \quad \text { or } \quad\left(I-C C^{\dagger}\right) B^{\dagger} D=0, \\
A\left(I-C^{\dagger} C\right)=0 \quad \text { or } \quad C C^{\dagger} B^{\dagger} D=0, \\
A\left(I-C^{\dagger} C\right)=0 \quad \text { or } \quad\left(I-C C^{\dagger}\right) B^{\dagger} D=0 .
\end{gathered}
$$

So if $\left(I-C C^{\dagger}\right) B^{\dagger} D \neq 0$, then $A C^{\dagger} C=0$ and $A\left(I-C^{\dagger} C\right)=0$. Thus $A=0$, which contradicts $A \neq 0$. Similarly, $A\left(I-C^{\dagger} C\right) \neq 0$ implies $B^{\dagger} D=0$. But, by (36), $B^{\dagger} D \neq 0$ since $D \neq 0$, which also leads to a contradiction. Hence

$$
\left(I-C C^{\dagger}\right) B^{\dagger} D=0, \quad A\left(I-C^{\dagger} C\right)=0 .
$$

Consequently, by (36) and (39),

$$
A=A C^{\dagger} C=A C^{\dagger} B^{\dagger} B C, \quad D=B B^{\dagger} D=B C C^{\dagger} B^{\dagger} D .
$$

Namely, $\mathscr{R}\left(A^{*}\right) \subseteq \mathscr{R}\left((B C)^{*}\right)$ and $\mathscr{R}(D) \subseteq \mathscr{R}(B C)$.

Take $B^{(1,2)}=\left[B^{\dagger}+\left(I-B^{\dagger} B\right) U\right] B B^{\dagger}$ and $C^{(1,2)}=C^{\dagger} C\left[C^{\dagger}+\right.$ $\left.V\left(I-C C^{\dagger}\right)\right]$, and put them into (29). Then, by (36) and (39),

$$
A C^{\dagger} C V\left(I-C C^{\dagger}\right)\left(I-B^{\dagger} B\right) U B B^{\dagger} D=0 .
$$

Since $A C^{\dagger} C=A \neq 0$ and $B B^{\dagger} D=D \neq 0$, then $\left(I-C C^{\dagger}\right)(I-$ $\left.B^{\dagger} B\right)=0$; by the arbitrariness of $U$ and $V$ and Lemma 3 , we have $\mathcal{N}(B) \subseteq \mathscr{R}(C)$.

(ii) $\Rightarrow$ (iii): By Theorem 5, Statement (iii) is obvious.

(iii) $\Rightarrow$ (ii): When replacing $C$ and $B$ in Statement (i) by $B C$ and $I$, respectively, we immediately get $\mathscr{R}\left(A^{*}\right) \subseteq \mathscr{R}\left((B C)^{*}\right)$ and $\mathscr{R}(D) \subseteq \mathscr{R}(B C)$ in view of the equivalence between (i) and (iii).

Now we will show $\mathcal{N}(B) \subseteq \mathscr{R}(C)$. Since $C^{(1,2)} B^{(1,2)} \in$ $(B C)\{1\}$, for any $C^{(1,2)} \in C\{1,2\}$ and $B^{(1,2)} \in B\{1,2\}$, by Lemma 1 ,

$$
\begin{aligned}
B C C^{(1)} B^{(1)} B C & =B C C^{(1)} C C^{(1)} B^{(1)} B B^{(1)} B C \\
& =B C C^{(1,2)} B^{(1,2)} B C=B C .
\end{aligned}
$$

Using Corollary 8, we have $\mathcal{N}(B) \subseteq \mathscr{R}(C)$.

The remainder is to discuss the situation under $A C^{\dagger} B^{\dagger} D$ having closed range. It is clear that (i) $\Rightarrow$ (iv). Following the process of the proof of (iv) $\Rightarrow$ (ii) in Theorem 5 , we can also turn out (iv) $\Rightarrow$ (ii) by the argument in the proof of (i) $\Rightarrow$ (ii).

Remark 10. Obviously Theorems 5 and 9 are equivalent. If either $B$ and $C$ is zero, then it must be false that $A C^{(1)} B^{(1)} D$ does not depend on the choice of $C^{(1)} \in C\{1\}$ and $B^{(1)} \in$ $B\{1\}$ except for $A=0$ or $D=0$. But it is always true that $A C^{(1,2)} B^{(1,2)} D$ does not depend on the choice of $C^{(1,2)} \in$ $C\{1,2\}$ and $B^{(1,2)} \in B\{1,2\}$ because 0 is the only $\{1,2\}$-inverse of 0 .

Finally we will investigate the situations with respect to $\{1,3\}$-inverses and $\{1,4\}$-inverses.

Theorem 11. Let nonzero operators $A \in \mathscr{L}(\mathscr{I}, \mathscr{H}), B \in$ $\mathscr{L}(\mathscr{J}, \mathscr{K}), C \in \mathscr{L}(\mathscr{I}, \mathscr{J})$, and $D \in \mathscr{L}(\mathscr{H}, \mathscr{K})$ have closed ranges. Then the first two statements below are equivalent. Suppose that $A C^{\dagger} B^{\dagger} D$ has closed range. Then the following statements are equivalent:

(i) $A C^{(1,3)} B^{(1,3)} D$ does not depend on the choice of $C^{(1,3)} \in$ $C\{1,3\}$ and $B^{(1,3)} \in B\{1,3\}$;

(ii) $\mathscr{R}\left(A^{*}\right) \subseteq \mathscr{R}\left(C^{*}\right)$ and $\mathscr{R}\left(\left(A C^{\dagger}\right)^{*}\right) \subseteq \mathscr{R}\left(B^{*}\right)$, or $\mathscr{R}(D) \subseteq \mathscr{N}\left(B^{*}\right)$ and $\mathcal{N}(B)=0$;

(iii) $\mathscr{R}\left(A C^{(1,3)} B^{(1,3)} D\right)$ does not depend on the choice of $C^{(1,3)} \in C\{1,3\}$ and $B^{(1,3)} \in B\{1,3\}$. 
Proof. (i) $\Rightarrow$ (ii): Clearly, $A C^{(1,3)} B^{(1,3)} D=A C^{\dagger} B^{\dagger} D$. Putting $C^{(1,3)}=C^{\dagger}+\left(I-C^{\dagger} C\right) V$ and $B^{(1,3)}=B^{\dagger}+\left(I-B^{\dagger} B\right) U$ into this equation leads to

$$
\begin{array}{r}
A\left(I-C^{\dagger} C\right) V B^{\dagger} D+A C^{\dagger}\left(I-B^{\dagger} B\right) U D \\
+A\left(I-C^{\dagger} C\right) V\left(I-B^{\dagger} B\right) U D=0
\end{array}
$$

for any $U$ and $V$. Then

$$
\begin{gathered}
A\left(I-C^{\dagger} C\right)=0 \quad \text { or } \quad B^{\dagger} D=0, \quad A C^{\dagger}\left(I-B^{\dagger} B\right)=0, \\
A\left(I-C^{\dagger} C\right)=0 \quad \text { or } \quad I-B^{\dagger} B=0 .
\end{gathered}
$$

Thus $A C^{\dagger}=A C^{\dagger} B^{\dagger} B$ and $A=A C^{\dagger} C$, or $B^{\dagger} D=0$ and $B^{\dagger} B=$ $I$. Hence (ii) is true.

(ii) $\Rightarrow$ (i): By Lemma 4, Statement (ii) implies $A C^{\dagger} B^{\dagger} B=$ $A C^{\dagger}$ and $A C^{\dagger} C=A$, or $B^{*} D=0$. So $B^{\dagger} D=0$. Since $\mathcal{N}(B)=$ 0 and $B\left(I-B^{\dagger} B\right)=0, B^{\dagger} B=I$. Now, by Lemma 1 ,

$$
\begin{aligned}
A C^{(1,3)} & B^{(1,3)} D \\
& =A\left(C^{\dagger}+\left(I-C^{\dagger} C\right) V\right)\left(B^{\dagger}+\left(I-B^{\dagger} B\right) U\right) D \\
& =A C^{\dagger}\left(B^{\dagger}+\left(I-B^{\dagger} B\right) U\right) D=A C^{\dagger} B^{\dagger} D .
\end{aligned}
$$

That is, Statement (i) is true.

Now consider the situation under $A C^{\dagger} B^{\dagger} D$ having closed range. Apparently, (i) implies (iii). We will show (iii) $\Rightarrow$ (ii).

Obviously, $\mathscr{R}\left(A C^{(1,3)} B^{(1,3)} D\right)=\mathscr{R}\left(A C^{\dagger} B^{\dagger} D\right)$ holds for every $C^{(1,3)} \in C\{1,3\}$ and $B^{(1,3)} \in B\{1,3\}$. Then $A C^{(1,3)} B^{(1,3)} D$ has closed range and therefore

$$
\left(A C^{\dagger} B^{\dagger} D\right)\left(A C^{\dagger} B^{\dagger} D\right)^{\dagger}=\left(A C^{(1,3)} B^{(1,3)} D\right)\left(A C^{(1,3)} B^{(1,3)} D\right)^{\dagger}
$$

Similar to the argument above, putting $C^{(1,3)}=C^{\dagger}+(I-$ $\left.C^{\dagger} C\right) V$ and $B^{(1,3)}=B^{\dagger}+\left(I-B^{\dagger} B\right) U$, the above equation leads to

$$
\begin{array}{r}
A\left(I-C^{\dagger} C\right) V B^{\dagger} D+A C^{\dagger}\left(I-B^{\dagger} B\right) U D \\
+A\left(I-C^{\dagger} C\right) V\left(I-B^{\dagger} B\right) U D=0
\end{array}
$$

and then (ii) can be reached.

Corollary 12. Let nonzero operators $A \in \mathscr{L}(\mathscr{I}, \mathscr{H}), B \in$ $\mathscr{L}(\mathscr{J}, \mathscr{K}), C \in \mathscr{L}(\mathscr{I}, \mathscr{J})$, and $D \in \mathscr{L}(\mathscr{H}, \mathscr{K})$ have closed ranges. Then $A C^{(1,3)} B^{(1,3)} D=0$ holds for every $C^{(1,3)} \in$ $C\{1,3\}$ and $B^{(1,3)} \in B\{1,3\}$ if and only if $\mathscr{R}\left(A^{*}\right) \subseteq \mathscr{R}\left(C^{*}\right)$, $\mathscr{R}\left(\left(A C^{\dagger}\right)^{*}\right) \subseteq \mathscr{R}\left(B^{*}\right)$, and $\mathscr{R}\left(B^{\dagger} D\right) \subseteq \mathcal{N}\left(A C^{\dagger}\right)$, or $\mathscr{R}(D) \subseteq$ $\mathcal{N}\left(B^{*}\right)$ and $\mathcal{N}(B)=0$.

Corollary 13 (see [10, Theorem 3.1]). Let nonzero operators $A \in \mathscr{L}(\mathscr{I}, \mathscr{H}), B \in \mathscr{L}(\mathscr{H}, \mathscr{K})$, and $C \in \mathscr{L}(\mathscr{H}, \mathscr{K})$ have closed ranges. Then the following statements are equivalent:

(i) the operator product $A B^{(1,3)} C$ does not depend on the choice of $B^{(1,3)} \in B\{1,3\}$;

(ii) $\mathscr{R}\left(A^{*}\right) \subseteq \mathscr{R}\left(B^{*}\right)$.
Note that $X \in A\{1,3\}$ if and only if $X^{*} \in A^{*}\{1,4\}$. Thus we have the next theorem by Theorem 11 and then the following corollaries.

Theorem 14. Let nonzero operators $A \in \mathscr{L}(\mathscr{I}, \mathscr{H}), B \in$ $\mathscr{L}(\mathscr{J}, \mathscr{K}), C \in \mathscr{L}(\mathscr{I}, \mathscr{J})$, and $D \in \mathscr{L}(\mathscr{H}, \mathscr{K})$ have closed ranges. Then the first two statements below are equivalent. Suppose that $A C^{\dagger} B^{\dagger} D$ has closed range. Then the following statements are equivalent:

(i) $A C^{(1,4)} B^{(1,4)} D$ does not depend on the choice of $C^{(1,4)} \epsilon$ $C\{1,4\}$ and $B^{(1,4)} \in B\{1,4\}$;

(ii) $\mathscr{R}(D) \subseteq \mathscr{R}(B)$ and $\mathscr{R}\left(B^{\dagger} D\right) \subseteq \mathscr{R}(C)$, or $\mathscr{R}\left(C^{*}\right) \subseteq$ $\mathcal{N}(A)$ and $\mathscr{N}\left(C^{*}\right)=0$

(iii) $\mathscr{R}\left(A C^{(1,4)} B^{(1,4)} D\right)$ does not depend on the choice of $C^{(1,4)} \in C\{1,4\}$ and $B^{(1,4)} \in B\{1,4\}$.

Corollary 15. Let nonzero operators $A \in \mathscr{L}(\mathscr{I}, \mathscr{H}), B \in$ $\mathscr{L}(\mathscr{J}, \mathscr{K}), C \in \mathscr{L}(\mathscr{I}, \mathscr{J})$ and $D \in \mathscr{L}(\mathscr{H}, \mathscr{K})$ have closed ranges. Then $A C^{(1,4)} B^{(1,4)} D=0$ holds for every $C^{(1,4)} \in C\{1,4\}$ and $B^{(1,4)} \in B\{1,4\}$ if and only if $\mathscr{R}(D) \subseteq \mathscr{R}(B), \mathscr{R}\left(B^{\dagger} D\right) \subseteq$ $\mathscr{R}(C)$, and $\mathscr{R}\left(B^{\dagger} D\right) \subseteq \mathcal{N}\left(A C^{\dagger}\right)$, or $\mathscr{R}\left(C^{*}\right) \subseteq \mathcal{N}(A)$ and $\mathcal{N}\left(C^{*}\right)=0$.

Corollary 16 (see [10, Theorem 3.4]). Let nonzero operators $A \in \mathscr{L}(\mathscr{I}, \mathscr{H}), B \in \mathscr{L}(\mathscr{H}, \mathscr{K})$, and $C \in \mathscr{L}(\mathscr{H}, \mathscr{K})$ have closed ranges. Then the following statements are equivalent:

(i) the operator product $A B^{(1,4)} C$ does not depend on the choice of $B^{(1,4)} \in B\{1,4\}$;

(ii) $\mathscr{R}(C) \subseteq \mathscr{R}(B)$.

\section{Range Inclusion Invariance Properties of Operator Product}

In the section, we will discuss the range inclusion invariance properties of the operator product involving generalized inverses. The following theorem is concerning the equivalent conditions of the topic.

Theorem 17. Let nonzero operators $A \in \mathscr{L}(\mathscr{J}, \mathscr{K}), B \in$ $\mathscr{L}(\mathscr{J}, \mathscr{I}), C \in \mathscr{L}(\mathscr{H}, \mathscr{I})$, and $D \in \mathscr{L}(\mathscr{M}, \mathscr{K})$ have closed ranges. Then the following statements are equivalent:

(i) $\mathscr{R}\left(A B^{(1)} C\right) \subseteq \mathscr{R}(D)$ holds for every $B^{(1)} \in B\{1\}$;

(ii) $\mathscr{R}\left(A B^{(1,2)} C\right) \subseteq \mathscr{R}(D)$ holds for every $B^{(1,2)} \in B\{1,2\}$;

(iii) $\mathscr{R}(A) \subseteq \mathscr{R}(D)$; or $\mathscr{R}(C) \subseteq \mathscr{R}(B), \mathscr{R}\left(A B^{\dagger} C\right) \subseteq \mathscr{R}(D)$ and $\mathscr{R}\left(A\left(I-B^{\dagger} B\right)\right) \subseteq \mathscr{R}(D)$.

Proof. (i) $\Rightarrow$ (ii): It is obvious. 

form:

(ii) $\Rightarrow$ (iii): By Lemma 2, we have the following matrix

$$
\begin{gathered}
A=\left(\begin{array}{cc}
A_{11} & A_{12} \\
0 & 0
\end{array}\right):\left(\begin{array}{c}
\mathscr{R}\left(B^{*}\right) \\
\mathcal{N}(B)
\end{array}\right) \longrightarrow\left(\begin{array}{c}
\mathscr{R}(A) \\
\mathcal{N}\left(A^{*}\right)
\end{array}\right), \\
B=\left(\begin{array}{cc}
B_{11} & 0 \\
0 & 0
\end{array}\right):\left(\begin{array}{c}
\mathscr{R}\left(B^{*}\right) \\
\mathcal{N}(B)
\end{array}\right) \longrightarrow\left(\begin{array}{c}
\mathscr{R}(B) \\
\mathcal{N}\left(B^{*}\right)
\end{array}\right), \\
C=\left(\begin{array}{cc}
C_{11} & 0 \\
C_{21} & 0
\end{array}\right):\left(\begin{array}{c}
\mathscr{R}\left(C^{*}\right) \\
\mathcal{N}(C)
\end{array}\right) \longrightarrow\left(\begin{array}{c}
\mathscr{R}(B) \\
\mathcal{N}\left(B^{*}\right)
\end{array}\right), \\
D=\left(\begin{array}{cc}
D_{11} & 0 \\
D_{21} & 0
\end{array}\right):\left(\begin{array}{c}
\mathscr{R}\left(D^{*}\right) \\
\mathcal{N}(D)
\end{array}\right) \longrightarrow\left(\begin{array}{c}
\mathscr{R}(A) \\
\mathcal{N}\left(A^{*}\right)
\end{array}\right), \\
B^{\dagger}=\left(\begin{array}{cc}
B_{11}^{-1} & 0 \\
0 & 0
\end{array}\right):\left(\begin{array}{c}
\mathscr{R}(B) \\
\mathcal{N}\left(B^{*}\right)
\end{array}\right) \longrightarrow\left(\begin{array}{c}
\mathscr{R}\left(B^{*}\right) \\
\mathcal{N}(B)
\end{array}\right), \\
D^{\dagger}=\left(\begin{array}{cc}
F^{-1} D_{11}^{*} & F^{-1} D_{21}^{*} \\
0 & 0
\end{array}\right):\left(\begin{array}{c}
\mathscr{R}(A) \\
\mathcal{N}\left(A^{*}\right)
\end{array}\right) \longrightarrow\left(\begin{array}{c}
\mathscr{R}\left(D^{*}\right) \\
\mathcal{N}(D)
\end{array}\right),
\end{gathered}
$$

where $B_{11}$ and $F=D_{11}^{*} D_{11}+D_{21}^{*} D_{21}$ are invertible in $\mathscr{L}\left(\mathscr{R}\left(B^{*}\right), \mathscr{R}(B)\right)$ and $\mathscr{L}\left(\mathscr{R}\left(D^{*}\right)\right)$, respectively. So, by Lemma 1 ,

$$
\begin{aligned}
B^{(1,2)} & =\left[B^{\dagger}+\left(I-B^{\dagger} B\right) X\right] B\left[B^{\dagger}+Y\left(I-B B^{\dagger}\right)\right] \\
& =\left(\begin{array}{cc}
B_{11}^{-1} & Y_{12} \\
X_{21} & X_{21} B_{11} Y_{12}
\end{array}\right),
\end{aligned}
$$

where $X=\left(X_{i j}\right), Y=\left(Y_{i j}\right), i, j=1,2$.

The inclusion $\mathscr{R}\left(A B^{(1,2)} C\right) \subseteq \mathscr{R}(D)$ is equivalent to $(I-$ $\left.D D^{\dagger}\right) A B^{(1,2)} C=0$; that is,

$$
\begin{aligned}
&(I-\left.D_{11} F^{-1} D_{11}^{*}\right) A_{11} B_{11}^{-1} C_{11}+\left(I-D_{11} F^{-1} D_{11}^{*}\right) A_{11} Y_{12} C_{21} \\
&+\left(I-D_{11} F^{-1} D_{11}^{*}\right) A_{12} X_{21} C_{11} \\
&+\left(I-D_{11} F^{-1} D_{11}^{*}\right) A_{12} X_{21} B_{11} Y_{12} C_{21}=0, \\
& D_{21} F^{-1} D_{11}^{*} A_{11} B_{11}^{-1} C_{11}+D_{21} F^{-1} D_{11}^{*} A_{11} Y_{12} C_{21} \\
&+ \\
&+D_{21} F^{-1} D_{11}^{*} A_{12} X_{21} C_{11} \\
&+ D_{21} F^{-1} D_{11}^{*} A_{12} X_{21} B_{11} Y_{12} C_{21}=0 .
\end{aligned}
$$

Thus, from the two equations above and the arbitrariness of $X_{i j}$ and $Y_{i j}$,

$$
\begin{gathered}
\left(I-D_{11} F^{-1} D_{11}^{*}\right) A_{12} X_{21} C_{11}=0, \\
\left(I-D_{11} F^{-1} D_{11}^{*}\right) A_{11} Y_{12} C_{21}=0, \\
\left(I-D_{11} F^{-1} D_{11}^{*}\right) A_{12} X_{21} B_{11} Y_{12} C_{21}=0, \\
D_{21} F^{-1} D_{11}^{*} A_{12} X_{21} C_{11}=0, \\
D_{21} F^{-1} D_{11}^{*} A_{11} Y_{12} C_{21}=0, \\
D_{21} F^{-1} D_{11}^{*} A_{12} X_{21} B_{11} Y_{12} C_{21}=0 .
\end{gathered}
$$

If $C_{21} \neq 0$, then, by (52) and (54),

$$
\begin{array}{r}
\left(I-D_{11} F^{-1} D_{11}^{*}\right) A_{1 j}=0, \quad D_{21} F^{-1} D_{11}^{*} A_{1 j}=0, \\
j=1,2 .
\end{array}
$$

Namely, $\left(I-D D^{\dagger}\right) A=\left(\begin{array}{cc}\left(I-D_{11} F^{-1} D_{11}^{*}\right) A_{11} & \left(I-D_{11} F^{-1} D_{11}^{*}\right) A_{12} \\ -D_{21} F^{-1} D_{11}^{*} A_{11} & -D_{21} F^{-1} D_{11}^{*} A_{12}\end{array}\right)=0$. Thus $\mathscr{R}(A) \subseteq \mathscr{R}(D)$.

If $C_{21}=0$, then $\left(I-B B^{\dagger}\right) C=0$ and $C_{11} \neq 0$. Therefore the former implies $\mathscr{R}(C) \subseteq \mathscr{R}(B)$ and the latter, by (51) and (53), implies

$$
\left(I-D_{11} F^{-1} D_{11}^{*}\right) A_{12}=0, \quad D_{21} F^{-1} D_{11}^{*} A_{12}=0 .
$$

Namely, $\left(I-D D^{\dagger}\right) A\left(I-B^{\dagger} B\right)=0$, and $\mathscr{R}\left(A\left(I-B^{\dagger} B\right)\right) \subseteq$ $\mathscr{R}(D)$.

It is clear that $\mathscr{R}\left(A B^{\dagger} C\right) \subseteq \mathscr{R}(D)$. As a result, we reach (iii).

(iii) $\Rightarrow$ (i): If $\mathscr{R}(A) \subseteq \mathscr{R}(D)$, then $\mathscr{R}\left(A B^{(1)} C\right) \subseteq \mathscr{R}(A) \subseteq$ $\mathscr{R}(D)$. Since $\mathscr{R}(C) \subseteq \mathscr{R}(B),\left(I-B B^{\dagger}\right) C=0$. Since $\mathscr{R}\left(A B^{\dagger} C\right) \subseteq \mathscr{R}(D)$ and $\mathscr{R}\left(A\left(I-B^{\dagger} B\right)\right) \subseteq \mathscr{R}(D), A B^{\dagger} C=D X$ for some $X$ and $A\left(I-B^{\dagger} B\right)=D Y$ for some $Y$. Then

$$
\begin{aligned}
A B^{(1)} C & =A\left(B^{\dagger}+\left(I-B^{\dagger} B\right) U+V\left(I-B B^{\dagger}\right)\right) C \\
& =A B^{\dagger} C+A\left(I-B^{\dagger} B\right) U C+A V\left(I-B B^{\dagger}\right) C \\
& =D(X+Y U C)
\end{aligned}
$$

and therefore $\mathscr{R}\left(A B^{(1)} C\right) \subseteq \mathscr{R}(D)$.

Remark 18. When $A, B, C$, and $D$ are matrices,

$$
\begin{aligned}
\mathscr{R}\left(A^{*} D^{\perp}\right) \subseteq \mathscr{R}\left(B^{*}\right) & \Longleftrightarrow D^{* \perp} A\left(I-B^{\dagger} B\right)=0 \\
& \Longleftrightarrow \mathscr{R}\left(A\left(I-B^{\dagger} B\right)\right) \subseteq \mathscr{N}\left(D^{* \perp}\right) \\
& =\mathscr{R}\left(D^{\perp}\right)^{\perp}=\mathscr{R}(D),
\end{aligned}
$$

where symbol $K^{\perp}$ denotes any matrix with $m$ rows such that $\mathscr{R}\left(K^{\perp}\right)$ coincides with the orthogonal complement of $\mathscr{R}(K)$ for $K \in \mathbb{C}^{m \times n}$ (see [9] for details). So by the argument in [9, page 97], Theorem 17(iii) is equipollent to [9, Theorem 2(ii)(c)] for matrices when $C \neq 0$.

In the following theorem, we state the range inclusion invariance involving $\{1,3\}$-inverse and $\{1,4\}$-inverse.

Theorem 19. Let nonzero operators $A \in \mathscr{L}(\mathscr{F}, \mathscr{K}), B \in$ $\mathscr{L}(\mathscr{J}, \mathscr{I}), C \in \mathscr{L}(\mathscr{H}, \mathscr{I})$, and $D \in \mathscr{L}(\mathscr{M}, \mathscr{K})$ have closed ranges. Then the following statements are equivalent:

(i) $\mathscr{R}\left(A B^{(1,3)} C\right) \subseteq \mathscr{R}(D)$ holds for every $B^{(1,3)} \in B\{1,3\}$;

(ii) $\mathscr{R}\left(A B^{\dagger} C\right) \subseteq \mathscr{R}(D), \mathscr{R}\left(A\left(I-B^{\dagger} B\right)\right) \subseteq \mathscr{R}(D)$.

Proof. (i) $\Rightarrow$ (ii): By Lemmas 1 and 2, we get

$$
\begin{array}{r}
B^{(1,3)}=B^{\dagger}+\left(I-B^{\dagger} B\right) X=\left(\begin{array}{cc}
B_{11}^{-1} & 0 \\
X_{21} & X_{22}
\end{array}\right), \\
\text { where } X=\left(X_{i j}\right), \quad i=1,2 .
\end{array}
$$


We know that $\mathscr{R}\left(A B^{(1,3)} C\right) \subseteq \mathscr{R}(D)$ is equivalent to $(I-$ $\left.D D^{\dagger}\right) A B^{(1,3)} C=0$; namely,

$$
\begin{aligned}
& \left(I-D_{11} F^{-1} D_{11}^{*}\right)\left[A_{11} B_{11}^{-1} C_{11}+A_{12} X_{21} C_{11}+A_{12} X_{22} C_{21}\right] \\
& \quad=0 \\
& D_{21} F^{-1} D_{11}^{*}\left[A_{11} B_{11}^{-1} C_{11}+A_{12} X_{21} C_{11}+A_{12} X_{22} C_{21}\right]=0 .
\end{aligned}
$$

Thus, from the two equations and the arbitrariness of $X_{i j}$,

$$
\begin{gathered}
\left(I-D_{11} F^{-1} D_{11}^{*}\right) A_{12} X_{21} C_{11}=0, \\
D_{21} F^{-1} D_{11}^{*} A_{12} X_{21} C_{11}=0, \\
\left(I-D_{11} F^{-1} D_{11}^{*}\right) A_{12} X_{22} C_{21}=0, \\
D_{21} F^{-1} D_{11}^{*} A_{12} X_{22} C_{21}=0 .
\end{gathered}
$$

If $C_{21} \neq 0$, then, by (62),

$$
\left(I-D_{11} F^{-1} D_{11}^{*}\right) A_{12}=0, \quad D_{21} F^{-1} D_{11}^{*} A_{12}=0 .
$$

If $C_{21}=0$, then $C_{11} \neq 0$ and therefore, by (61), we also get (63). Hence, from (63), $\left(I-D D^{\dagger}\right) A\left(I-B^{\dagger} B\right)=0$; namely, $\mathscr{R}\left(A\left(I-B^{\dagger} B\right)\right) \subseteq \mathscr{R}(D)$.

It is clear that $\mathscr{R}\left(A B^{\dagger} C\right) \subseteq \mathscr{R}(D)$. Consequently, (ii) is reached.

(ii) $\Rightarrow$ (i): Since $\mathscr{R}\left(A B^{\dagger} C\right) \subseteq \mathscr{R}(D)$ and $\mathscr{R}\left(A\left(I-B^{\dagger} B\right)\right) \subseteq$ $\mathscr{R}(D), A B^{\dagger} C=D X$ for some $X$ and $A\left(I-B^{\dagger} B\right)=D Y$ for some $Y$. Then

$$
\begin{aligned}
A B^{(1,3)} C & =A\left(B^{\dagger}+\left(I-B^{\dagger} B\right) U\right) C \\
& =A B^{\dagger} C+A\left(I-B^{\dagger} B\right) U C=D(X+Y U C)
\end{aligned}
$$

and therefore $\mathscr{R}\left(A B^{(1,3)} C\right) \subseteq \mathscr{R}(D)$.

Concerning $\{1,4\}$-inverses, we have the following result.

Theorem 20. Let nonzero operators $A \in \mathscr{L}(\mathscr{J}, \mathscr{K}), B \in$ $\mathscr{L}(\mathscr{J}, \mathscr{I}), C \in \mathscr{L}(\mathscr{H}, \mathscr{I})$ and $D \in \mathscr{L}(\mathscr{M}, \mathscr{K})$ have closed ranges. Then the following statements are equivalent:

(i) $\mathscr{R}\left(A B^{(1,4)} C\right) \subseteq \mathscr{R}(D)$ holds for every $B^{(1,4)} \in B\{1,4\}$;

(ii) $\mathscr{R}(A) \subseteq \mathscr{R}(D)$, or $\mathscr{R}(C) \subseteq \mathscr{R}(B)$ and $\mathscr{R}\left(A B^{\dagger} C\right) \subseteq$ $\mathscr{R}(D)$.

Proof. (i) $\Rightarrow$ (ii): By Lemmas 1 and 2,

$$
\begin{array}{r}
B^{(1,4)}=B^{\dagger}+X\left(I-B B^{\dagger}\right)=\left(\begin{array}{cc}
B_{11}^{-1} & X_{12} \\
0 & X_{22}
\end{array}\right), \\
\text { where } X=\left(X_{i j}\right), \quad i=1,2 .
\end{array}
$$

The condition $\mathscr{R}\left(A B^{(1,4)} C\right) \subseteq \mathscr{R}(D)$ is equivalent to $(I-$ $\left.D D^{\dagger}\right) A B^{(1,4)} C=0$; namely,

$$
\begin{aligned}
& \left(I-D_{11} F^{-1} D_{11}^{*}\right)\left[A_{11} B_{11}^{-1} C_{11}+A_{11} X_{12} C_{21}+A_{12} X_{22} C_{21}\right] \\
& \quad=0 \\
& D_{21} F^{-1} D_{11}^{*}\left[A_{11} B_{11}^{-1} C_{11}+A_{11} X_{12} C_{21}+A_{12} X_{22} C_{21}\right]=0 .
\end{aligned}
$$

Thus, from the two equations and the arbitrariness of $X_{i j}$,

$$
\begin{array}{r}
\left(I-D_{11} F^{-1} D_{11}^{*}\right) A_{1 i} X_{i 2} C_{21}=0, \\
D_{21} F^{-1} D_{11}^{*} A_{1 i} X_{i 2} C_{21}=0, \\
i=1,2 .
\end{array}
$$

If $C_{21} \neq 0$, then, by the four equations above,

$$
\begin{array}{r}
\left(I-D_{11} F^{-1} D_{11}^{*}\right) A_{1 i}=0, \\
D_{21} F^{-1} D_{11}^{*} A_{1 i}=0, \\
i=1,2 .
\end{array}
$$

Hence $\left(I-D D^{\dagger}\right) A=0$; namely, $\mathscr{R}(A) \subseteq \mathscr{R}(D)$.

If $C_{21}=0$, then $\left(I-B B^{\dagger}\right) C=0$ and $\mathscr{R}(C) \subseteq \mathscr{R}(B)$.

It is clear that $\mathscr{R}\left(A B^{\dagger} C\right) \subseteq \mathscr{R}(D)$. Consequently, (ii) is reached.

(ii) $\Rightarrow$ (i): Since $\mathscr{R}\left(A B^{\dagger} C\right) \subseteq \mathscr{R}(D)$ and $\mathscr{R}(C) \subseteq \mathscr{R}(B)$, $A B^{\dagger} C=D X$ for some $X$ and $C=B Y$ for some $Y$. Then

$$
\begin{aligned}
A B^{(1,4)} C & =A\left(B^{\dagger}+U\left(I-B B^{\dagger}\right)\right) C \\
& =A B^{\dagger} C+A U\left(I-B B^{\dagger}\right) C=D X
\end{aligned}
$$

and therefore $\mathscr{R}\left(A B^{(1,4)} C\right) \subseteq \mathscr{R}(D)$.

If $\mathscr{R}(A) \subseteq \mathscr{R}(D)$, then clearly $\mathscr{R}\left(A B^{(1,4)} C\right) \subseteq \mathscr{R}(D)$.

\section{Conflict of Interests}

The authors declare that there is no conflict of interests regarding the publication of this paper.

\section{Acknowledgments}

Xiaoji Liu and Miao Zhang were supported by the Guangxi Natural Science Foundation (2013GXNSFAA019008), the Key Project of Education Department of Guangxi (201202ZD031), Project supported by the National Science Foundation of China (11361009), and Science Research Project 2013 of the China-ASEAN Study Center (Guangxi Science Experiment Center) of Guangxi University for Nationalities.

\section{References}

[1] A. Ben-Israel and T. N. E. Greville, Generalized Inverses: Theory and Applications, CMS Books in Mathematics/Ouvrages de Mathématiques de la SMC, 15, Springer, New York, 2nd edition, 2003. 
[2] C. R. Rao and S. K. Mitra, Generalized Inverse of Matrices and Its Applications, John Wiley \& Sons, New York, NY, USA, 1971.

[3] R. Penrose, "A generalized inverse for matrices," Proceedings of Cambridge Philosophical Society, vol. 51, pp. 406-413, 1955.

[4] G. Wang, Y. Wei, and S. Qiao, Generalized Inverses: Theory and Computations, Science Press, Beijing, China, 2004.

[5] J. K. Baksalary and R. Kala, "Range invariance of certain matrix products," Linear and Multilinear Algebra, vol. 14, no. 1, pp. 8996, 1983.

[6] J. K. Baksalary and T. Mathew, "Rank invariance criterion and its application to the unified theory of least squares," Linear Algebra and Its Applications, vol. 127, pp. 393-401, 1990.

[7] J. K. Baksalary and T. Pukkila, "A note on invariance of the eigenvalues, singular values, and norms of matrix products involving generalized inverses," Linear Algebra and Its Applications, vol. 165, pp. 125-130, 1992.

[8] J. K. Baksalary and O. M. Baksalary, "An invariance property related to the reverse order law," Linear Algebra and Its Applications, vol. 410, pp. 64-69, 2005.

[9] J. Groß and Y. Tian, "Invariance properties of a triple matrix product involving generalized inverses," Linear Algebra and Its Applications, vol. 417, no. 1, pp. 94-107, 2006.

[10] Z. Xiong and Y. Qin, "Invariance properties of an operator product involving generalized inverses," Electronic Journal of Linear Algebra, vol. 22, pp. 694-703, 2011.

[11] D. S. Djordjević and N. Č. Dinčić, "Reverse order law for the Moore-Penrose inverse," Journal of Mathematical Analysis and Applications, vol. 361, no. 1, pp. 252-261, 2010. 


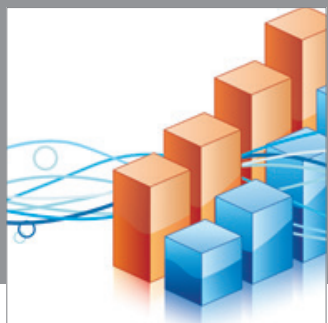

Advances in

Operations Research

mansans

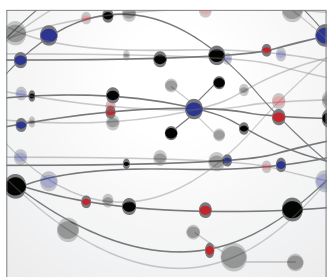

The Scientific World Journal
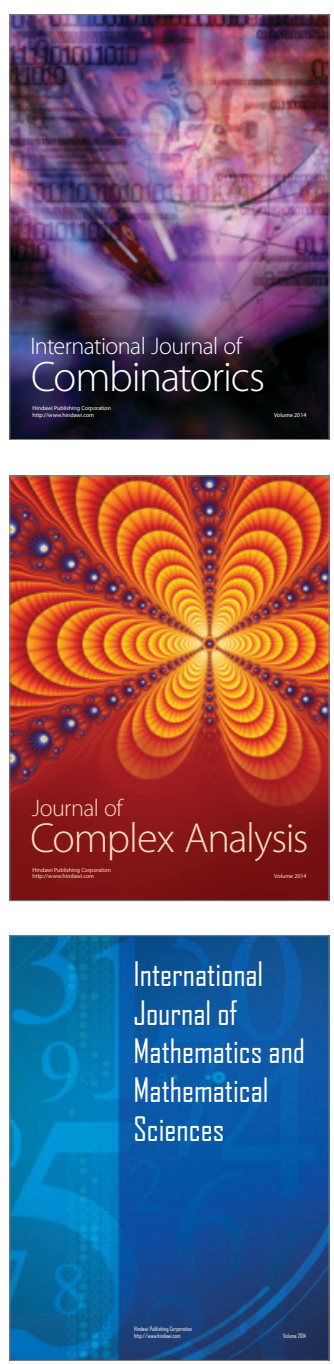
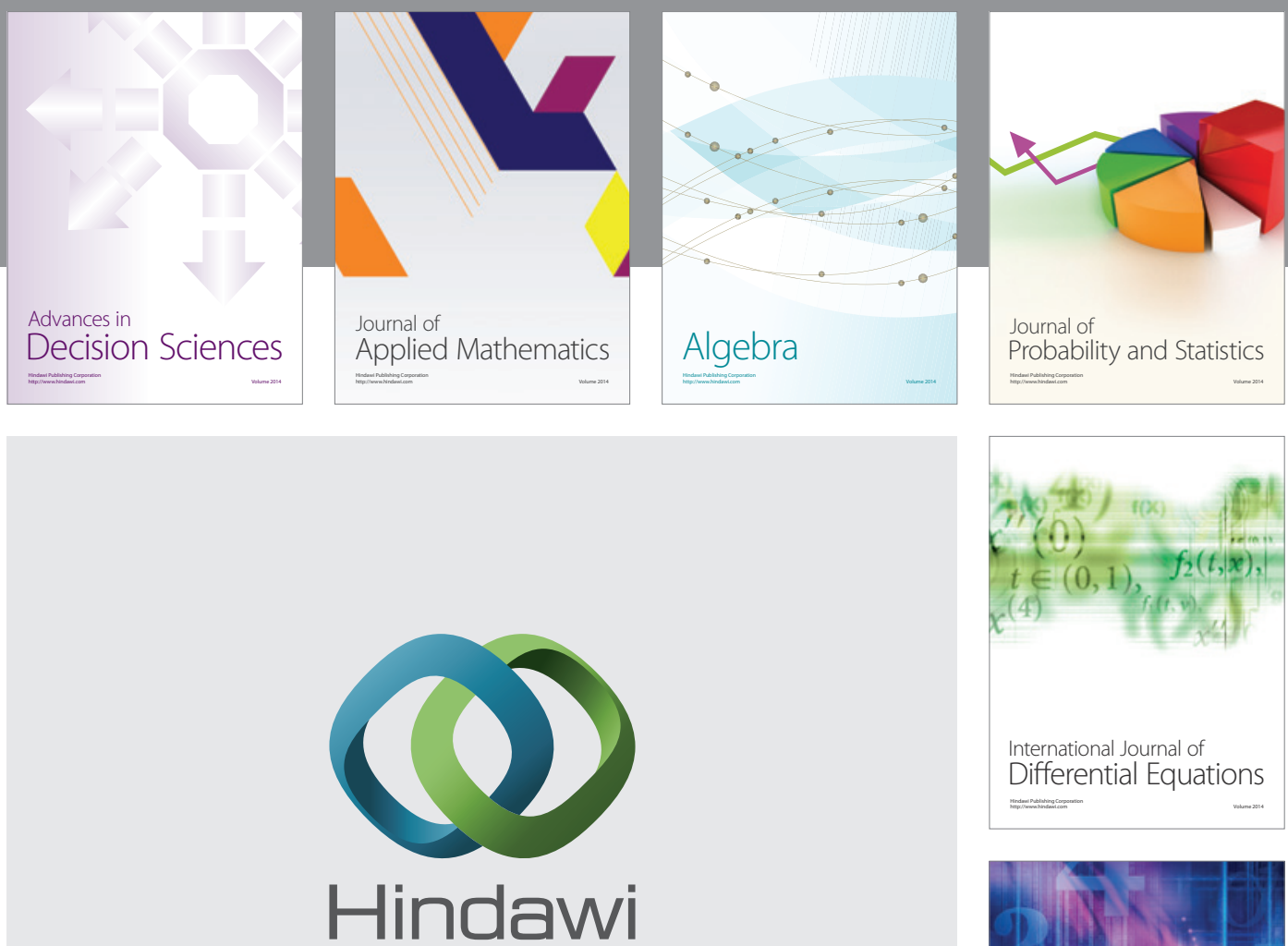

Submit your manuscripts at http://www.hindawi.com
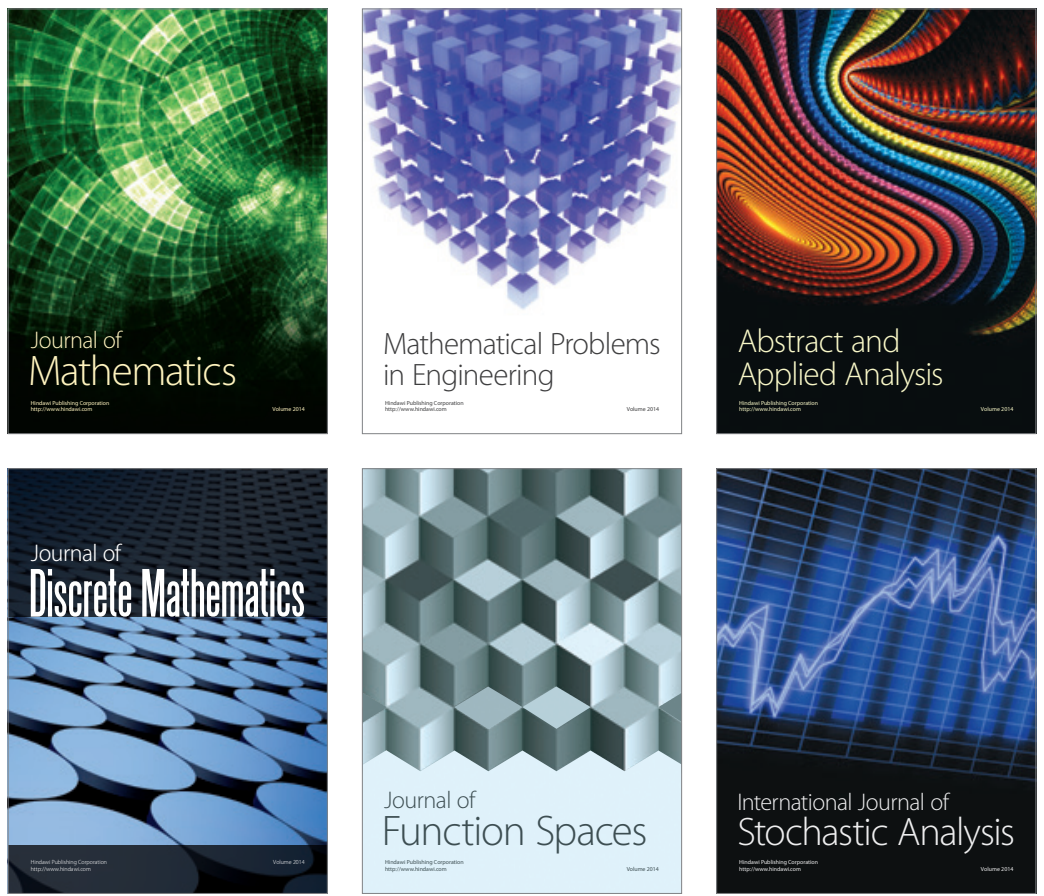

Journal of

Function Spaces

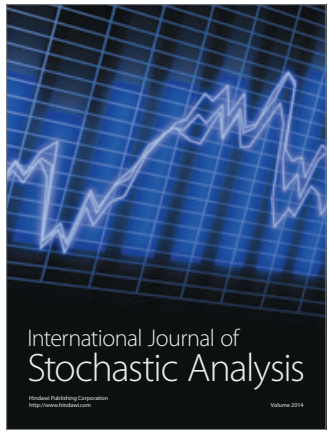

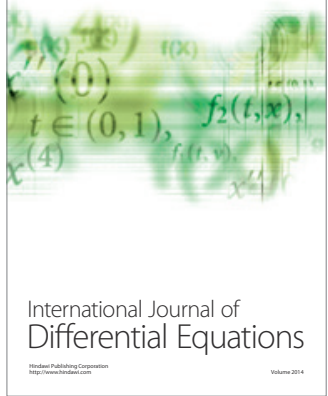
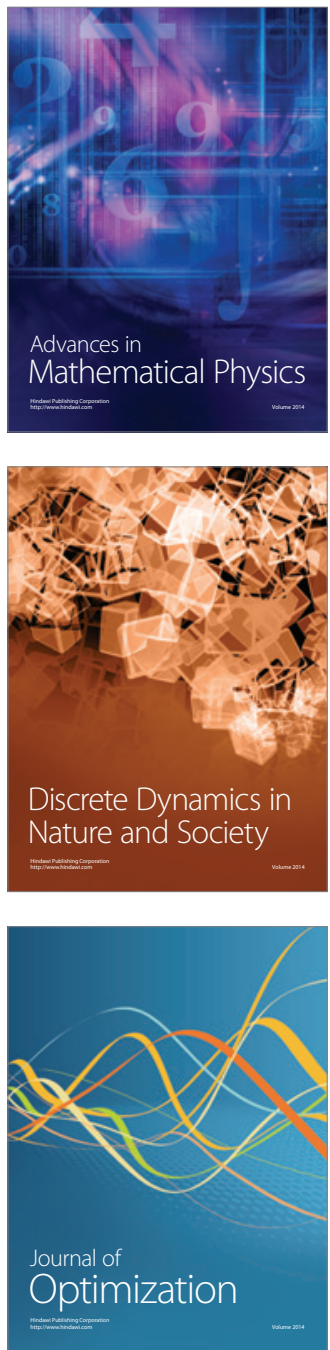